\title{
New Space Leaky Wave Spectrum Components on the Slotline
}

\author{
Ján Zehentner, Jan Macháč \\ Czech Technical University, Technická 2, 16627 Prague 6, Czech Republic \\ Tel +420 2 24352273, Fax +420 2 24355865, E-mail zeh@mbox.cesnet.cz
}

\begin{abstract}
Surface and space leakage of power deteriorates the operation of circuits. On the other hand space leaky waves enable the design of specific radiators. In this paper we therefore investigate the potential of the slotline to radiate into space. We confirm the findings of earlier studies and extend them to higher order modes. Leakage into the third and fifth higher order space leaky waves of the second class, with odd symmetry of the electric field within the slot, and leakage into the second higher order space leaky waves of first and also second class, with even symmetry of the electric field within the slot, are interesting new findings. We also present the measured and calculated radiation patterns of a slotline antenna operating with the first higher order space leaky wave of the first class.
\end{abstract}

\section{INTRODUCTION}

Planar transmission lines such as the microstrip line, strip line, and also uniplanar lines such as the coplanar waveguide, coplanar strips and the slotline are subjects of ongoing interest for researchers. Their new behavioural aspects are gradually being revealed. This paper deals with the slotline and introduces new components of its space leaky wave spectrum.

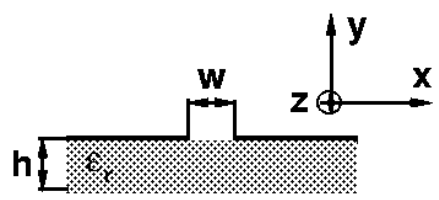

Fig. 1 Cross-section of the slotline

The slotline, the cross-section of which is shown in Fig. 1, has been known and applied in circuits for more than thirty years. Now, when the propagation characteristics of the dominant and first higher order bound waves as well as the surface leaky waves on the slotline are known [1-3], millimeter-wave antenna applications have stimulated investigation of space leaky waves. Sheen and Lin [4,5] recently published a study dealing with the so-called surface-wave-like mode and the first higher order mode, which we term the first higher order bound wave and the space leaky wave of the first class.
On the basis of this knowledge, we began our own investigation of space leaky waves on the slotline by means of the method of moments in the spectral domain. The crucial point is the deformation of the path of integration in the complex plane of the spectral variable. In the study of space leakage, we now have to take into account not only the poles related with the surface waves on a grounded dielectric slab but also the branch cut and branch point when choosing the integration path. We calculated the propagation constants of the first higher order bound mode and of the space leaky wave of the first class associated with this bound mode on a laterally unbounded slotline. Our data departed from those given in Fig. 7 and Fig. 3 in [5], within the range of normalized frequencies $\left(\mathrm{h} / \lambda_{0}\right)$ from 0.02 to 0.04 , by less than $4 \%$ in the phase constant and by less than $0.7 \%$ in the leakage constant. Since the difference between the published characteristics and our own are acceptably low, we applied this procedure in the whole succeeding analysis. We categorise particular waves as even and odd, according to the distribution of the transversal electric field component with regard to a plane of symmetry placed in the middle of the slot.

There is already much knowledge about the propagation constant of the dominant, 1st higher order and 3rd higher order bound wave $[1,3,6]$, as well as about the propagation constant of the space leaky mode of the first class associated with the 1st higher order bound wave on the slotline. We now present the phase constant for the 2nd higher order bound wave in this paper. Moreover our new findings concern the dispersion characteristics of the space leaky wave of the second class associated with the 1st and 3rd higher order bound wave, and the space leaky wave characteristics of the first and second class associated with the 2nd higher order bound wave. This means that there is leakage of energy into higher order space leaky waves, with odd or even symmetry of the electric field within the slot. In addition, we report our experience of designing a slotline-leaky wave antenna, its feeding, and its measured and calculated radiation pattern.

\section{SPACE LEAKY WAVES OF THE 2ND CLASS WITH ODD ELECTRIC FIELD SYMMETRY}

The slotline dispersion equation provides not only the space leaky wave solution of the first class 


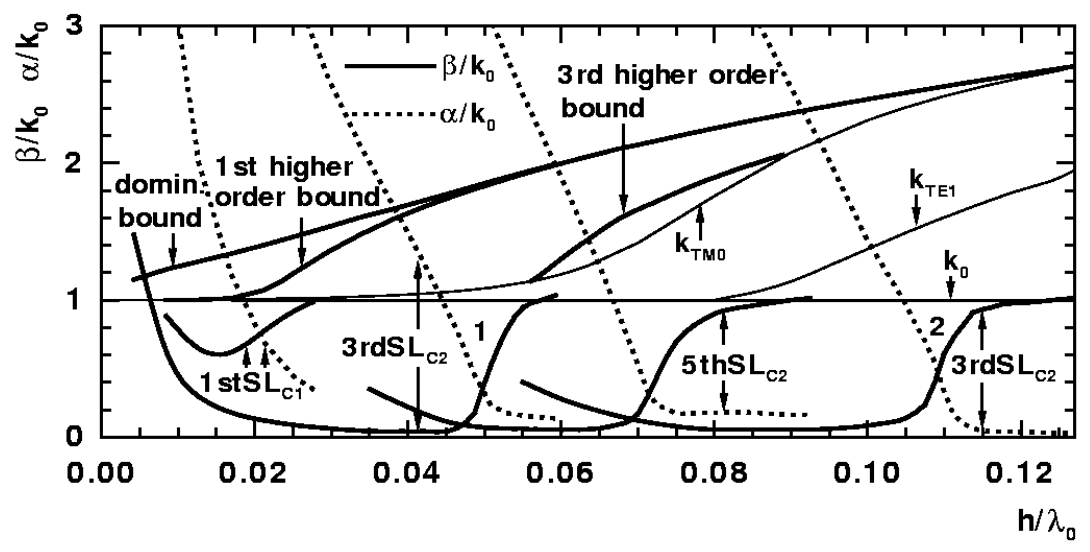

Fig. 2 Dispersion characteristics of the dominant, the 1st and 3rd higher-order bound waves, the 1st space leaky wave of class 1 , and of the $3 \mathrm{rd}$ and 5 th space leaky waves of class 2 on the slotline with $\varepsilon_{\mathrm{r}}=10.8, \mathrm{w}=20 \mathrm{~mm}, \mathrm{~h}=1.27 \mathrm{~mm}$

introduced in [5], but also a series of solutions of the space leaky wave of the second class presented here now. The number of such solutions is sequentially placed along the frequency axis. Only three of these solutions $\left(3 \mathrm{rdSL}_{\mathrm{C} 2}\right.$ no. 1,2 and $\left.5 \mathrm{thSL}_{\mathrm{C} 2}\right)$ are plotted in Fig. 2 where, for the sake of completeness, the solution $1 \mathrm{stSL} \mathrm{C}_{1}$ is also shown. The normalized phase $\left(\beta / \mathrm{k}_{0}\right)$ and leakage $\left(\alpha / \mathrm{k}_{0}\right)$ constants of the $3 \mathrm{rdSL}_{\mathrm{C} 2}$ solution were calculated with 2 and also with 4 basis functions in the $\mathrm{x}$ and $\mathrm{z}$ direction (Fig. 1) with almost no influence on the values of the constants. For the first branch of $3 \mathrm{rdSL}_{\mathrm{C} 2}$ only the pole of the $\mathrm{TM}_{0}$ surface wave was accounted for, while for the second branch of $3 \mathrm{rdSL}_{\mathrm{C} 2}$ the pole of the $\mathrm{TE}_{1}$ surface wave was also considered at frequencies higher than $\mathrm{TE}_{1}$ cut-off. The $5 \mathrm{thSL}_{\mathrm{C} 2}$ solution was calculated with 3 basis functions in the $\mathrm{x}$ and $\mathrm{z}$ direction. The transversal electric field component $\mathrm{E}_{\mathrm{x}}$ within the slot for the first branch of the $3 \mathrm{rdSL}_{\mathrm{C} 2}$ mode resembles $\mathrm{E}_{\mathrm{x}}$ of the $3 \mathrm{rd}$ higher order bound mode, as is shown in Fig. 3. We checked the same feature for the second branch of the $3 \mathrm{rdSL}_{\mathrm{C} 2}$. The $3 \mathrm{rdSL}_{\mathrm{C} 2}$ and $5 \mathrm{thSL}_{\mathrm{C} 2}$ solutions are physically meaningful at higher frequencies, where the phase constant exhibits a considerable increase and the leakage constant is reasonably low.

\section{SPACE LEAKY WAVES OF THE 1ST AND 2ND CLASS WITH EVEN ELECTRIC FIELD SYMMETRY}

A space leaky wave of the second class was first discovered on a suspended microstrip, and its excitation was confirmed by a rather less convincing resonant frequency measurement in [7]. The longitudinal current component on the strip has even symmetry, which is an analogy of our $E_{x}$ or an equivalent longitudinal magnetic current component on the slotline. We have revealed the propagation constant of this type of space leaky wave on a slotline.

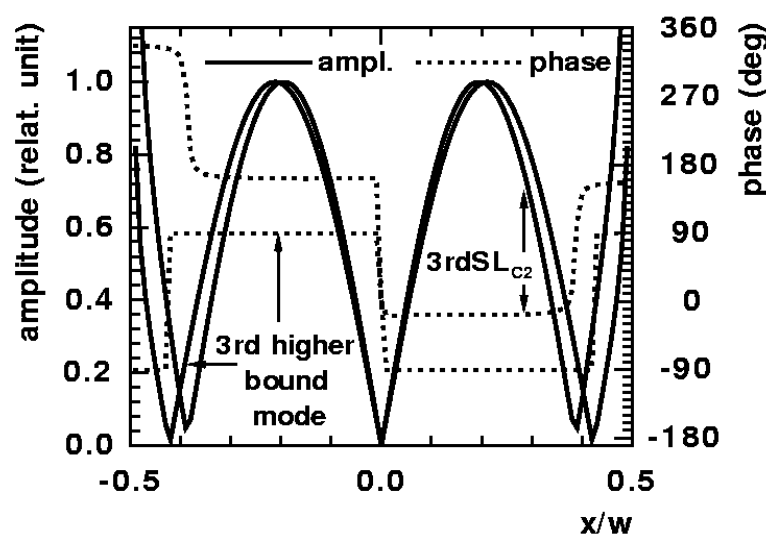

Fig. 3 Magnitude and phase of the transversal component of electric field $\mathrm{E}_{\mathrm{x}}$ within the slot for the 3rd higher-order bound mode and for the 3 rd space leaky wave of class 2 on the slotline from Fig. 2 when $\mathrm{h} / \lambda_{0}=0.0565$

The normalized propagation constant of the 2nd higher order bound wave, phase and leakage constant of the 2 nd space leaky wave of first class and also the

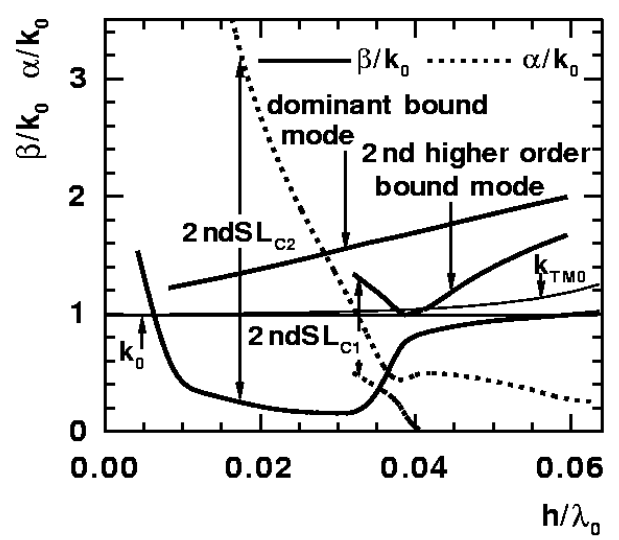

Fig. 4 Dispersion characteristics of the dominant, 2nd higher order bound wave, and the 2nd space leaky waves of class 1 and 2 on the slotline from Fig. 2

second class are plotted in Fig. 4. The dispersion 
characteristics of the $2 \mathrm{ndSL}_{\mathrm{C} 1}$ were hard to find, since their presence depends sensitively on the permittivity of the substrate and slotwidth. There is, however, a very narrow frequency band within which the phase constant is lower than $\mathrm{k}_{0}$. The practical importance of this wave on the slotline with the given parameters is limited. Fig. 5 provides a detailed view of the part of the characteristics from Fig. 4 where the $2 \mathrm{ndSL}_{\mathrm{C} 1}$ wave is physical. Here we can see the standard transition from the 2 nd higher order bound wave to the corresponding surface leaky wave involving a nonphysical real solution [8]. The surface leaky wave is physical for the given slotline at two frequency bands where $\mathrm{k}_{0}<\beta<\mathrm{k}_{\mathrm{TM}}$. This wave can propagate simultaneoustly with the space leaky wave $2 \mathrm{ndSL}_{\mathrm{Cl}}$. On the other hand, the propagation constant of the 2 ndSL $_{\mathrm{C} 2}$ wave has a typical pattern and a reasonably wide interval of physical meaningfulness as is shown by one branch of its dispersion characteristic plotted in Fig. 4. Fig. 6 shows that the distributions of the $E_{x}$ field component of the second higher order bound wave and the 2nd space leaky wave of class 1 and 2 , within the slot, are similar. It follows from [7] and from our own findings that not only a field with odd symmetry, but also fields with even symmetry can leak into space.

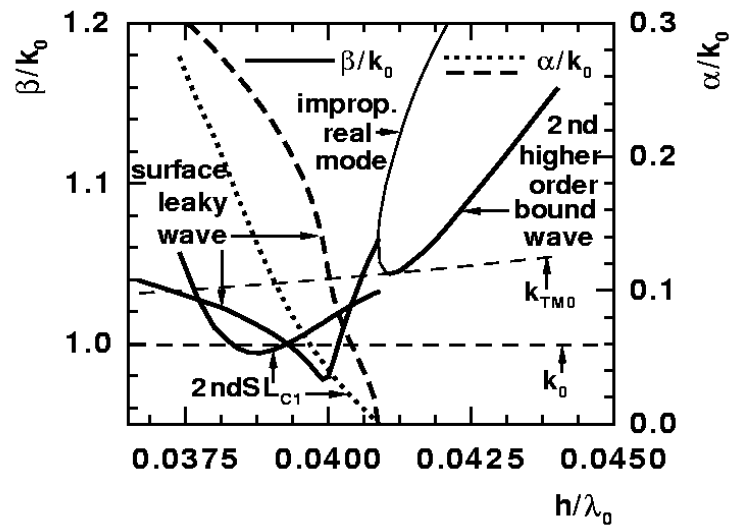

Fig. 5 Detail of dispersion characteristics from Fig. 4.

The dispersion characteristics of higher order bound waves touch the $\mathrm{k}_{\mathrm{TM} 0}$ curve on the high frequency side, Fig. 2. We have confirmed the standard transitions from the proper real solution of the dominant/higher order bound wave to a complex solution of the corresponding higher order surface leaky wave already described in [8].

\section{A SLOTLINE LEAKY-WAVE ANTENNA}

We designed a slotline leaky-wave antenna operating with the first space leaky wave of the first class. Similar work was already done in [5]. In our case, the microstrip line fed the antenna. Such feeding enables simpler coupling with the coaxial output of the generator, and according to [5] it has a better impedance match than the $\mathrm{CPW}$ feed. A plexiglass substrate and cross-sectional dimensions allowed excitation of the first space leaky wave of the first class from 3 to $4.5 \mathrm{GHz}$. We determined the normalized

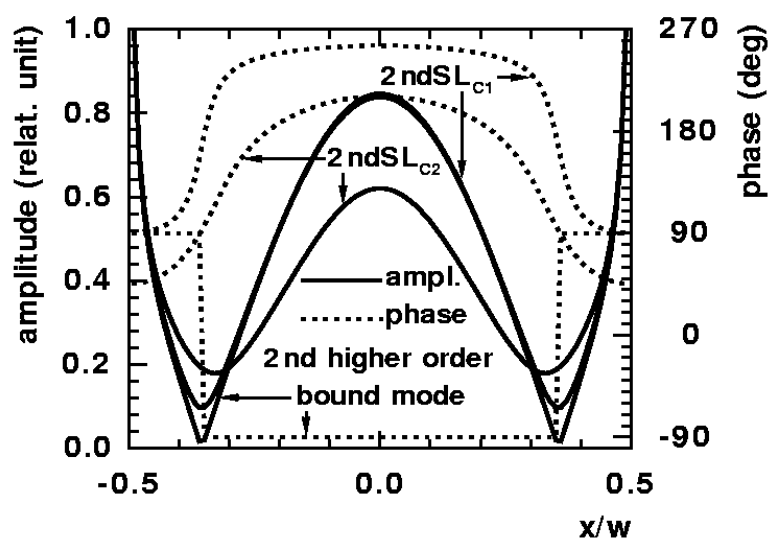

Fig. 6 Magnitude and phase of the transversal component of electric field $\mathrm{E}_{\mathrm{x}}$ within the slot for the 2nd space leaky wave of class 1 and 2 at $h / \lambda_{0}=0.0389$, and for the 2 nd higher order bound wave at $\mathrm{h} / \lambda_{0}=0.0444$ on the slotline from Fig. 2 .

phase constant of the $1 \mathrm{stSL} \mathrm{C}_{\mathrm{C} 1}$ wave computed from the measured resonances set up on the slotline. Measured and calculated $\beta / \mathrm{k}_{0}$ for the slotline on the plexiglass compare well, as shown in Fig. 7. The antenna layout went through several changes resulting from gradual measurements and simulation. It turned out that the shorter the slotlength, the better is the suppression of the side-lobes. Conversely the leakage constant determines the shortest admissible slotlength. The microstripslotline junction was adjusted experimentally to reflection loss lower than $-10 \mathrm{~dB}$ from 3.45 to $4.15 \mathrm{GHz}$ and with the minimum $-18 \mathrm{~dB}$ at $3.82 \mathrm{GHz}$. The antenna was terminated by a narrow slotline, as shown in the inset of Fig. 8, on which the first higher order bound wave cannot propagate. The simultaneous propagation of the $1 \mathrm{st}$ higher order bound wave and the $1 \mathrm{stSL}_{\mathrm{C} 1}$ wave is a problem, since until now it has not been possible to separate them

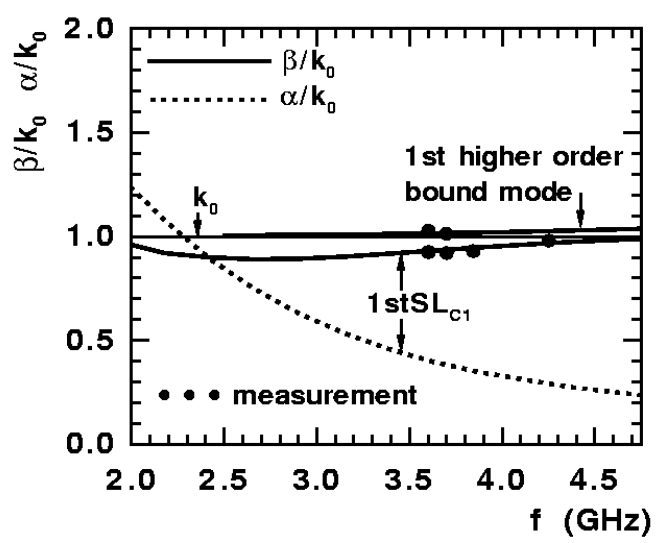

Fig. 7 Normalized phase and leakage constant of the $1 \mathrm{stSL}_{\mathrm{C} 1}$ wave on the slotline with $\varepsilon_{\mathrm{r}}=2.6, \mathrm{w}=40 \mathrm{~mm}, \mathrm{~h}=3.1 \mathrm{~mm}$ 


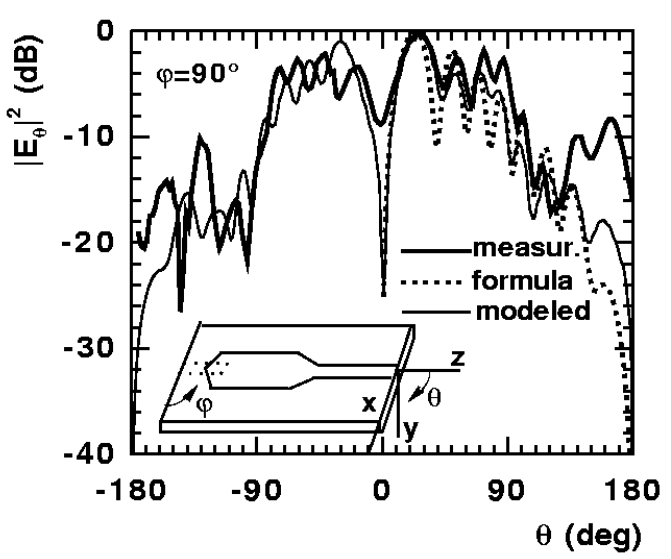

Fig. 8 Calculated superposition of the radiation patterns of the $1 \mathrm{st}$ higher order bound wave and the $1 \mathrm{stSL}_{\mathrm{C} 1}$ wave, and the measured radiation pattern of the slotline antenna at $3.82 \mathrm{GHz}$. The line parameters are from Fig. 6.

satisfactorily. The measured and modeled radiation patterns and the superposition of the 1st higher bound wave and the $1 \mathrm{stSL}_{\mathrm{C} 1}$ wave of the first class radiation patterns, calculated as the field of an ideal travellingwave line source [9], are compared in Fig. 8 at 3.82 GHz. We used Zeland IE3D software for the modeling. The patterns fit each other relatively well in the endfire direction.

\section{CONCLUSIONS}

The dominant and higher order bound waves, and also the space leaky waves of the first or second class, can propagate simultaneously on the slotline. Which constituent wave excites will depend on the frequency, permittivity and dimensions of the slotline, and on its feeding structure. Such multi-wave propagation causes undesirable couplings and cross-talk in a circuit, while splitting of the radiation pattern of an antenna may occur. In the latter case, the antenna must be designed carefully, to allow the occurrence of only the desired space leaky wave. Generally speaking, a space leaky wave can propagate at lower frequencies than the surface leaky wave does. The operation areas of these two types of leaky waves are either completely separated or may overlap.

We have identified new solutions of the slotline dispersion equation belonging to the space leaky waves of the first and second class, associated with both odd and even distribution of the transversal electric field component within the slot. These waves refine the spectrum of radiated waves. They are difficult to separate, since their field distribution resembles the fields of the bound waves. We have demonstrated the excitation of the first space leaky wave on the slotline. Some useful experience as regards the layout of the antenna and its feed is given. This paper contributes to the ongoing exploration of the behaviour of the slotline.

\section{ACKNOWLEDGMENTS}

This work has been supported by the Grant Agency of the Czech Republic under project 102/00/0025 „Planar circuits for mw and mmw technology" and by the research programme J04/98 212300014 „Information and Communication Technology", and was carried out on the SP-2 computer at the Joint Supercomputer Center of the Czech Technical University, University of Chemical Technology and IBM Prague.

\section{REFERENCES}

[1] H. Shigesawa, M. Tsuji, A. A. Oliner: Simultaneous Propagation of Bound and Leaky Dominant Modes on Printed-Circuit Lines: A New General Effect, IEEE Trans. Microwave Theory Tech., Vol. MTT-43, 1995, No. 12, pp. 3007-3019.

[2] M. Tsuji, N. Yahata, H. Shigesawa: Significant Contribution of Improper-Real Solution to the Fields Excited by a Practical Source in Printed-Circuit Transmission Lines, 1999 IEEE MTT-S IMS Digest, Anaheim, CA, Vol. 3, pp. 57-60.

[3] J. Zehentner, J. Macháč, M. Migliozzi: Upper Cutoff Frequency of the Bound Wave and New Leaky Wave on the Slotline, IEEE Trans. Microwave Theory Tech., Vol. MTT-46, 1998, No. 4, pp. 378-386.

[4] J.-W. Sheen, Y.-D. Lin: Surface-Wave-Like Mode in Slot Line, IEEE Microwave and Guided Wave Letters, Vol. 6 , 1996, No. 7, pp. 259-261.

[5] J.-W. Sheen, Y.-D. Lin: Propagation Characteristics of the Slotline First Higher Order Mode, IEEE Trans. Microwave Theory Tech., Vol. MTT-46, 1998, No. 11, pp. 1774-1781.

[6] K. Mejsnar: Higher-Order Modes on Slot Lines, 10th European Microwave Conference Proceedings, pp. 327330, Warszawa

[7] C.-K. C. Tzuang, C.-C. Lin: Fast-Wave Resonance by Space-Wave Leaky Mode Carrying Dominant-Mode-Like Currents, IEEE Trans. Microwave Theory Tech., Vol. MTT-46, 1998, No. 12, pp. 2444-2449.

[8] A. A. Oliner, D. R. Jackson: On spectral gaps at the transition between bound and leaky modes, Proceedings of the 1995 International Symposium on Electromagnetic Theory, pp. 764-766, St. Petersburg, Russia, May 1995.

[9] C. H. Walter: Traveling Wave Antennas, McGraw-Hill Book Comp., New York 1965. 